\title{
Nonexistence of Global Solutions of Systems of Time Fractional Differential equations posed on the Heisenberg group
}

\author{
Mokhtar Kirane ${ }^{1}$ and alrazi abdeljabbar ${ }^{1}$ \\ ${ }^{1}$ Khalifa University of Science and Technology
}

November 19, 2021

\begin{abstract}
We first consider the nonlinear time fractional diffusion equation $D^{\wedge}\{1+\alpha\} u+D^{\wedge} \beta u-[?]-\{H\} u=|u|^{\wedge} p$ posed on the Heisenberg group $\mathrm{H}$, where $1<\mathrm{p}$ is a positive real nimber to be specified later; $\mathrm{D}^{\wedge} \delta_{-}\{0 \mid \mathrm{t}\}$ is the Liouville-Caputo derivative of order $\delta$. For $0<\alpha<1,0<\beta$ [?] 1. This equation interpolates the heat equation and the wave equation with the linear damping $\mathrm{D}^{\wedge} \beta_{-}\{0 \mid t\} \mathrm{u}$. We present the Fujita exponent for blow-up. Then establish sufficient conditions ensuring non-existence of local solutions. We extend the analysis to the case of the system $D^{\wedge}\{1+\alpha\} u+D^{\wedge} \beta u-[?]_{-}\{H\} u=|v|^{\wedge} q D^{\wedge}\{1+\delta\}+D^{\wedge} \gamma v-[?]_{-}\{H\} v=|u|^{\wedge} p$. Our method of proof is based on the nonlinear capacity method.
\end{abstract}

\section{Hosted file}

paper.pdf available at https://authorea.com/users/298176/articles/546132-nonexistence-ofglobal-solutions-of-systems-of-time-fractional-differential-equations-posed-on-theheisenberg-group 